\title{
Mesure des énergies de rupture interfaciale : problématique et exemples de résultats d'essais de gonflement-décollement
}

\author{
Michel Dupeux ${ }^{\mathrm{a}}$ \\ Laboratoire de Thermodynamique et Physico-Chimie Métallurgiques (CNRS/INPG/UJF), ENSEEG, BP 75, \\ 38402 Saint Martin d'Hères Cedex, France
}

Reçu le 10 juin 2003, accepté le 7 novembre 2003

\begin{abstract}
Résumé - La résistance mécanique des interfaces entre deux matériaux différents constitue un problème critique, tant d'un point de vue fondamental que d'un point de vue appliqué. De fait, la décohésion interfaciale est un mécanisme fréquent de défaillance des composants multimatériaux ou des pièces revêtues, d'usage de plus en plus courant dans les technologies avancées actuelles. Un certain nombre de considérations générales sont exposées sur les phénomènes de décohésion et la mécanique de la rupture interfaciale, dans l'objectif d'en déduire les critères qui assurent une validité optimale aux résultats des essais mécaniques de mesure d'adhérence. Parmi les techniques expérimentales existantes, l'accent est mis sur celles qui permettent une détermination fiable de l'énergie de rupture interfaciale. Le cas de l'essai de gonflement-décollement est développé. Les résultats obtenus par cette technique sur plusieurs systèmes couches/substrats sont présentés et commentés.
\end{abstract}

Mots clés : Interfaces / rupture interfaciale / adhérence / énergie d'adhérence / essai de gonflementdécollement

\begin{abstract}
Measurement of interfacial crack propagation energy-related problems and examples of results of buldge-and-blister test. The mechanical strength of interfaces between two different materials is a critical point, as well on a fundamental as on a practical aspect. Indeed, interfacial debonding is a frequent failure mechanism for multimaterial parts or coatings, which are more and more in use in advanced technologies. Several general considerations are introduced about interfacial debonding phenomena and interfacial fracture mechanics, aiming at criteria to define the most relevant adhesion mechanical tests, able to provide reproducible and physically significant results. Among the available experimental techniques for mechanical adhesion measurement, those which provide reliable values of interfacial crack propagation energy are emphasized. The example of bulge and blister testing is developed in more details. Results obtained by this technique on various film/substrate systems are presented and commented on.
\end{abstract}

Key words: Interfaces / interfacial fracture / adhesion / adhesion energy / bulge and blister test

\section{Introduction}

L'optimisation de l'adéquation matériau/fonction dans les applications technologiques de pointe conduit à l'utilisation de plus en plus fréquente de «multimatériaux », « matériaux hybrides », composites, assemblages brasés ou collés, revêtements, multicouches structuraux ou fonctionnels. Selon l'application visée, différentes caractéristiques fonctionnelles sont attendues des interfaces solide/solide que contiennent ces pièces mécaniques ou composants monobloc hétérogènes. Mais dans tous les cas, la condition minimale nécessaire au

a Auteur correspondant : michel.dupeux@ltpcm.inpg.fr maintien de l'intégrité du matériau est une résistance suffisante des interfaces à la rupture par décohésion.

La résistance mécanique des interfaces entre deux matériaux différents constitue donc un problème critique, tant d'un point de vue appliqué que d'un point de vue fondamental, car toutes les difficultés que pose sa caractérisation sont loin d'être résolues.

\section{Généralités}

\subsection{Adhésion et adhérence}

Un numéro spécial de la Revue de Métallurgie Science et Génie des Matériaux [1] constitue une bonne 


\section{Nomenclature}

\begin{tabular}{|c|c|c|}
\hline$a$ & rayon (cercle) ou demi-côté (carré) d'une membrane en cours de gonflement & $\mu \mathrm{m}$ \\
\hline$E$ & module de Young & $\mathrm{MPa}$ \\
\hline$G c$ & $\begin{array}{l}\text { valeur critique du taux de restitution d'énergie lors de la propagation } \\
\text { d'une fissure dans un matériau homogène }\end{array}$ & J.m ${ }^{-2}$ \\
\hline$G_{i c}$ & $\begin{array}{l}\text { valeur critique du taux de restitution d'énergie lors de la propagation } \\
\text { d'une fissure interfaciale }\end{array}$ & J.m ${ }^{-2}$ \\
\hline$h$ & flèche au centre d'une cloque en cours de gonflement & $\mu \mathrm{m}$ \\
\hline$i$ & nombre imaginaire pur & \\
\hline$K$ & facteur d'intensité de contraintes complexe $K=K_{\mathrm{I}}+i K_{\mathrm{II}}$ & \\
\hline$K_{\mathrm{I} c}, K_{\mathrm{II} c}$ & $\begin{array}{l}\text { valeurs critiques du facteur d'intensité de contraintes provoquant la propagation } \\
\text { d'une fissure en mode I (resp. mode II) }\end{array}$ & MPa.m $\mathrm{m}^{1 / 2}$ \\
\hline$p$ & pression appliquée (essai de gonflement-décollement) & $\mathrm{Pa}$ \\
\hline$(r, \theta)$ & coordonnées polaires & \\
\hline$t$ & épaisseur d'un revêtement & $\mu \mathrm{m}$ \\
\hline$W_{\text {adhésion }}$ & énergie d'adhésion interfaciale, dite « énergie de Dupré » & J.m $m^{-2}$ \\
\hline$\alpha$ & premier coefficient de Dundurs & (ss. dim.) \\
\hline$\beta$ & second coefficient de Dundurs & (ss. dim.) \\
\hline$\gamma_{\mathrm{A}}, \gamma_{\mathrm{B}}$ & énergies de surface des solides A ou B & J.m ${ }^{-2}$ \\
\hline$\gamma_{\mathrm{AB}}$ & énergie des liaisons chimiques A-B à travers l'interface A/B & J.m ${ }^{-2}$ \\
\hline$\varepsilon$ & coefficient d'hétérogénéité élastique & (ss. dim.) \\
\hline$\psi, \psi^{*}$ & angles de mixité modale & $\operatorname{rad}$ \\
\hline$\lambda$ & ordre d'une singularité de contraintes & (ss. dim.) \\
\hline$\mu_{\mathrm{A}}, \mu_{\mathrm{B}}$ & module d'élasticité au cisaillement des matériaux A ou B & $\mathrm{GPa}$ \\
\hline$\nu_{\mathrm{A}}, \nu_{\mathrm{B}}$ & coefficients de Poisson des matériaux A ou B & (ss. dim.) \\
\hline$\sigma, \tau$ & $\begin{array}{l}\text { composantes de contrainte normale (resp. tangentielle) } \\
\text { sur une facette matérielle donnée }\end{array}$ & $\mathrm{MPa}$ \\
\hline$\sigma_{0}$ & contrainte résiduelle dans un revêtement sur substrat & $\mathrm{MPa}$ \\
\hline
\end{tabular}

introduction bibliographique sur ce sujet. On s'accorde pour définir intuitivement l'adhérence d'une interface comme sa résistance mécanique à la décohésion. Cette adhérence résulte de la convolution de deux types de facteurs :

- la contribution thermodynamique de l'énergie d'adhésion, c'est-à-dire le gain d'énergie de liaison chimique par unité de surface résultant de la constitution (en conditions thermodynamiquement réversibles) des liaisons chimiques $\mathrm{A}-\mathrm{B}$ entre les deux matériaux $\mathrm{A}$ et $\mathrm{B}$ adjacents, en remplacement des deux surfaces libres A et B pré-existantes :

$$
W_{\text {adhésion }}=\gamma_{\mathrm{A}}+\gamma_{\mathrm{B}}-\gamma_{\mathrm{AB}}
$$

Cette énergie dite «de Dupré » est de l'ordre du Joule par $\mathrm{m}^{2}$. Elle est influencée naturellement par la nature chimique des matériaux A et B, mais également par la structure (cristalline ou amorphe) du joint interfacial, par la présence éventuelle de contaminants ou de couches de réaction ( interphases ) dans l'interface.

- la contribution mécanique de l'énergie dissipée par un certain nombre de processus dissipatifs irréversibles activés lors de la mise en ouvre d'un essai mécanique d'adhérence, qui provoque une décohésion par propagation d'une fissure interfaciale. Ces processus très divers sont sensibles notamment à la rugosité de l'interface, à la présence et à la nature d'un champ de contraintes résiduelles dans les deux matériaux, à l'activation de plasticité ou de micro-fissuration secondaire en tête de fissure, au mouillage du fond de fissure par le milieu extérieur... Le résultat de la mesure de l'énergie de propagation d'une fissure interfaciale s'avère donc la plupart du temps d'un ordre de grandeur bien supérieur à l'énergie d'adhésion

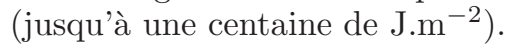

Ces deux types de contributions sont couplés par des effets encore incomplètement décrits et modélisés [2]. À défaut d'un paramètre physico-mécanique intrinsèque plus évident, l'énergie nécessaire à la rupture d'une l'interface apparaît donc comme la caractéristique la plus pertinente de l'adhérence interfaciale. Sa mesure est un enjeu majeur pour l'ensemble du champ d'application des multimatériaux. Cependant, la plupart des essais mécaniques classiques ne permettent pas d'accéder de manière simple à cette grandeur, car ils sollicitent l'interface via une action couplée des deux matériaux adjacents. 


\subsection{Effets de l'orientation de la sollicitation appliquée et de l'hétérogénéité}

Quelques études de la littérature ont montré, au moins sur des systèmes modèles, qu'un facteur prépondérant pour l'énergie de rupture interfaciale est l'orientation de la sollicitation mécanique appliquée pour propager la fissure interfaciale [3-6]. Le résultat obtenu lors de la mesure de l'énergie de propagation de fissure peut varier d'au moins un ordre de grandeur selon que l'interface est sollicitée en contrainte normale ou tangentielle. La mécanique de la rupture classique intègre bien le fait que les valeurs critiques des facteurs d'intensité de contrainte sont supérieures en mode II ou III à celle du mode I, mais ceci y est sans grande conséquence pratique : dans un matériau homogène, la fissure peut le plus souvent s'orienter spontanément par rapport au tenseur de contraintes local qui la fait progresser, de manière à se propager en mode I avec un minimum de coût énergétique. Et la valeur critique du facteur d'intensité de contrainte en mode I, $K_{\text {Ic }}$, suffit alors à caractériser la tenue à la fissuration du matériau.

Dans le cas présent d'une fissure d'interface, le plan de la fissure est au contraire imposé, dans un champ de contraintes dont l'orientation dépend à la fois des efforts appliqués à l'éprouvette et de l'hétérogénéité des propriétés des deux composants du bi-matériau. En effet, la simple différence des constantes d'élasticité engendre des perturbations locales du champ de contraintes appliquées, nécessaires pour rendre compatibles les déformations des matériaux accolés à l'interface. Ces effets de couplage élastique de deux matériaux $\mathrm{A}$ et $\mathrm{B}$ différents sont caractérisés classiquement par les paramètres de Dundurs [7] :

$$
\begin{aligned}
& \alpha=\frac{\mu_{\mathrm{A}}\left(1-\nu_{\mathrm{B}}\right)-\mu_{\mathrm{B}}\left(1-\nu_{\mathrm{A}}\right)}{\mu_{\mathrm{A}}\left(1-\nu_{\mathrm{B}}\right)+\mu_{\mathrm{B}}\left(1-\nu_{\mathrm{A}}\right)} \\
& \beta=\frac{1}{2}\left[\frac{\mu_{\mathrm{A}}\left(1-2 \nu_{\mathrm{B}}\right)-\mu_{\mathrm{B}}\left(1-2 \nu_{\mathrm{A}}\right)}{\mu_{\mathrm{A}}\left(1-\nu_{\mathrm{B}}\right)+\mu_{\mathrm{B}}\left(1-\nu_{\mathrm{A}}\right)}\right]
\end{aligned}
$$

d'où se déduit un troisième paramètre d'hétérogénéité utile :

$$
\varepsilon=\frac{1}{2 \pi} \ln \left(\frac{1-\beta}{1+\beta}\right)
$$

L'hétérogénéité, qui modifie le champ de contraintes local en tête de fissure, doit donc être prise en compte pour déterminer, au-delà de la simple géométrie des efforts appliqués à l'éprouvette, quel mode de sollicitation exact provoque la propagation de cette fissure.

\section{3 Éléments de mécanique de la rupture interfaciale [8]}

Au voisinage de l'extrémité d'une fissure d'interface, la manière la plus simple de décrire les champs de contraintes fait appel à des fonctions de la variable complexe $(x+i y)$ ou $r e^{i \theta}$ dans le repère défini sur la figure 1. En raison des effets de couplage élastique évoqués ci-dessus, il n'est pas possible de traiter de manière

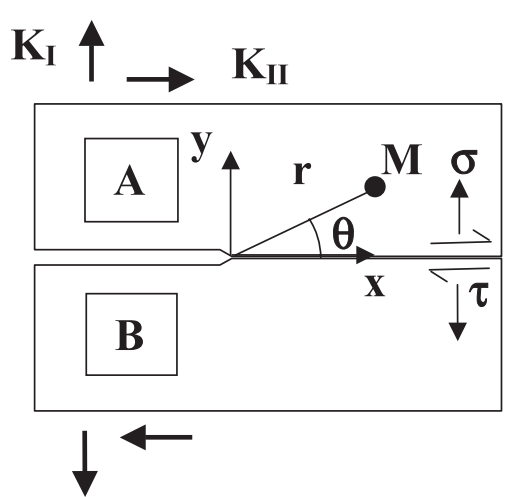

Fig. 1. Sollicitations sur une fissure interfaciale et conventions de coordonnées (la fissure est en $x<0$; l'indice A correspond au matériau situé en $y>0$ ).

indépendante les modes I, II et III comme on peut le faire en milieu homogène. Si le mode III demeure indépendant, en revanche, les modes I et II apparaissent couplés dans la partie réelle et la partie imaginaire d'un facteur d'intensité de contrainte complexe $K=K_{\mathrm{I}}+i K_{\mathrm{II}}$. Sur une facette interfaciale $(\theta=0)$ au voisinage de l'apex de la fissure, les composantes de contraintes normale et tangentielle sont alors couplées, et données (en élasticité isotrope pure) par l'expression complexe :

$$
(\sigma+i \tau)_{r, \theta=0}=\frac{K \cdot r^{i \varepsilon}}{\sqrt{2 \pi r}}
$$

où $\varepsilon$ est le paramètre d'hétérogénéité défini par (4). Les proportions de sollicitation en mode I et en mode II qui s'appliquent sur la fissure sont quantifiées à l'aide de l'angle de mixité modale $\psi$ :

$$
\psi=\operatorname{Arg}(K)=\operatorname{Arctg}\left(K_{\mathrm{II}} / K_{\mathrm{I}}\right)
$$

La valeur de $\psi$ ainsi définie présente l'inconvénient de dépendre du choix des unités de longueur utilisées. Aussi peut-on lui préférer l'angle $\psi^{*}$, qui ne dépend plus des unités de longueur, mais dépend encore de la distance $r$ à la pointe de fissure. $\psi^{*}$ représente le rapport contrainte tangentielle sur contrainte normale que subit une facette interfaciale située dans la zone singulière [9] :

$$
\begin{aligned}
\psi * & =\operatorname{Arctg}(\tau / \sigma)=\operatorname{Arg}\left(K . r^{i \varepsilon}\right) \\
& =\operatorname{Arctg}\left[\operatorname{Im}\left(K r^{i \varepsilon}\right) / \operatorname{Re}\left(K r^{i \varepsilon}\right)\right]
\end{aligned}
$$

Un examen attentif de l'expression (5) permet de s'apercevoir que le champ de contraintes est non seulement divergent à l'approche de la pointe de fissure (point singulier), mais aussi que la mixité modale locale $\psi^{*}$ évolue de plus en plus rapidement dans cette région dite de «K-dominance », entraînant la présence d'oscillations de contraintes et de déplacements ; celles-ci n'ont heureusement que peu de conséquences pratiques, car dans la plupart des cas réels, elles n'apparaissent qu'en dessous d'une distance à la singularité de l'ordre des distances interatomiques, donc sans signification physique. 

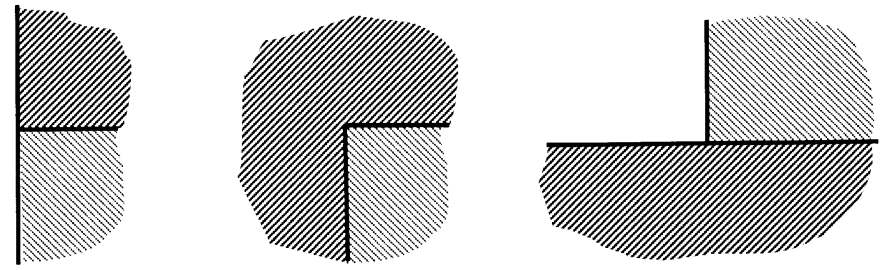

Fig. 2. Représentations schématiques de divers cas d'arêtes singulières géométriques et matérielles.

Une modélisation analytique ou numérique peut permettre de connaître ce champ de contraintes dans la région de $\mathrm{K}$-dominance dans une situation donnée de fissuration interfaciale, par exemple sur une éprouvette d'essai d'adhérence [10]. La détermination des conditions critiques de chargement $K_{c}=K_{\mathrm{I} c}+i K_{\mathrm{II} c}$ provoquant la propagation de la fissure permet alors de déterminer l'énergie de propagation $G_{i c}$, définie par :

$$
\left\|K_{c}\right\|^{2}=G_{i c}\left[\frac{1-\nu_{\mathrm{A}}^{2}}{2 E_{\mathrm{A}}}+\frac{1-\nu_{\mathrm{B}}^{2}}{2 E_{\mathrm{B}}}\right]^{(-1)} \cdot \frac{1}{\left(1-\beta^{2}\right)}
$$

Par analogie avec la relation $K_{c}^{2}=G_{c} . E$ applicable dans le cas d'un matériau homogène, le facteur multiplicatif de $G_{i c}$ dans l'expression (8) définit un « module de Young équivalent $»$ de l'interface pour ce qui concerne la propagation de fissure.

L'introduction de la plasticité complique naturellement ce formalisme [8].

\subsection{Problème de l'amorçage de la fissure}

Dans la quasi-totalité des cas réels de structure multimatériaux, toute fissure interfaciale préexistante est soigneusement évitée avant la mise en service de la pièce. Les défaillances par rupture interfaciale doivent donc être amorcées par les sollicitations en service à partir de sites d'amorçage préférentiels. Ceux-ci sont fournis par les arêtes dièdres de jonction des surfaces internes des différents matériaux entre elles ou avec les surfaces libres de la pièce (cf. schémas de la Fig. 2). Ces arêtes constituent fréquemment des lignes singulières des champs de contraintes, mais où la singularité est moins « sévère » qu'au front d'une fissure interfaciale : ceci signifie schématiquement que les composantes du champ des contraintes au voisinage de la singularité sont décrites par une série dont le terme principal est de la forme :

$$
\sigma_{i j}(r, \theta)=K_{\lambda \cdot} f_{i j}(\theta) \cdot r^{-\lambda}+\ldots
$$

expression dans laquelle le paramètre $\lambda$, appelé ordre de la singularité, prend une valeur comprise entre 0 et 0,5 , le cas limite $\lambda=0,5$ correspondant à une fissure parfaite [11]. On remarquera que le facteur d'intensité de contraintes $K_{\lambda}$ défini par l'expression (9) a la dimension de $\mathrm{MPa} \cdot \mathrm{m}^{\lambda}$, et n'est donc pas physiquement directement comparable ni au $K_{c}$ de l'expression (8), ni aux valeurs classiques de $K_{\text {Ic }}$ de la littérature pour les matériaux homogènes.
Diverses études récentes [12,13] permettent d'envisager désormais la formulation d'un critère d'amorçage d'une fissure à partir d'une telle singularité « faible » [10] . Dans la suite de ce texte, nous accorderons cependant une place privilégiée aux techniques qui permettent d'éviter les aléas de l'étape d'amorçage de la fissure interfaciale.

\section{Techniques de mesure de l'énergie d'adhérence}

\subsection{Critères de qualification d'un essai d'adhérence}

On peut attendre deux types de résultats d'un essai d'adhérence, selon le contexte dans lequel on l'effectue :

- soit on souhaite une simple comparaison à des valeurs de référence de la résistance mécanique en service d'une interface intégrée dans la structure multimatériaux dont elle fait partie : il conviendra alors de procéder à des essais dans des conditions de géométrie et de sollicitation aussi proches que possible de celles de la pièce réelle dans sa situation d'intégrité initiale; - soit on recherche des informations absolues, quantifiables, d'ordre physico-chimique et physicomécanique sur l'adhérence interfaciale elle-même, et c'est alors l'énergie critique de propagation de fissure interfaciale qui apparaît comme le paramètre pertinent à mesurer, indépendamment des conditions d'amorçage de la fissure.

\subsubsection{Caractérisation de l'adhérence en service}

L'éprouvette aussi représentative que possible comprendra dans ce cas des interfaces incluant les sites d'amorçage constitués par les arêtes singulières, tels que le processus d'élaboration les a produits. L'accroissement des sollicitations réalistes sur cette «pièce-éprouvette » fait apparaître un seuil de défaillance par décohésion, conditionné autant par l'étape d'amorçage de la fissure interfaciale que par sa propagation, et dont la validation ne pourra se passer d'un traitement statistique. En effet, en pratique, l'amorçage le long des arêtes dièdres est toujours soumis aux aléas de la taille du plus « sévère » des défauts présents, lié à la qualité de la préparation de la pièce ou de l'éprouvette.

Par exemple, dans cet esprit, la norme NF EN ISO 2819 recommande des techniques d'appréciation semi-quantitative de l'adhérence des revêtements électrolytiques sur leurs substrats métalliques.

\subsubsection{Caractérisation absolue de l'adhérence}

Ce type de mesure d'énergie de propagation de fissure interfaciale exigera alors des éprouvettes spécifiques,

- comportant une amorce de fissure préexistante de longueur connue, pour éviter les aléas de l'étape d'amorçage; 
- se prêtant à une modélisation précise du champ de contraintes afin de pouvoir relier à chaque instant de l'essai les conditions critiques de propagation de la fissure à une valeur numérique de $G_{i c}$, avec prise en compte d'éventuelles contraintes résiduelles, et calcul de la valeur de la mixité modale;

- conçues de manière à ce que, dans le cas où l'un des matériaux du couple a un comportement fragile, la fissure interfaciale n'aie pas tendance à quitter l'interface pour dévier en mode I vers ce matériau (signe approprié de la mixité modale par rapport à la disposition des matériaux $\mathrm{A}$ et $\mathrm{B}$ );

- et enfin, l'énergie de propagation mesurée dépendant fortement de la valeur de la mixité modale utilisée, il sera nécessaire, pour caractériser complètement la réponse d'une interface donnée, de disposer d'une gamme d'essais capables de couvrir la plage $0 \leq \psi \leq$ $\pi / 2$.

Les essais rencontrés dans la littérature diffèrent également selon qu'ils s'appliquent à des bi-matériaux massifs ou à des systèmes couche/substrat. Cette dernière classe de systèmes constitue à l'heure actuelle un champ d'application privilégié des essais d'adhérence, compte tenu du développement croissant des revêtements fonctionnels et des couches minces. Notons qu'il est souvent possible de transformer un système couche/substrat en bi-matériau massif en rajoutant sur la couche, symétriquement au substrat, un massif rapporté fixé à l'aide d'un adhésif structural dont l'adhérence doit être au moins aussi bonne que celle de l'interface couche/substrat à tester.

Quelques articles passent en revue diverses techniques de mesure de l'adhérence interfaciale appartenant aux catégories définies ci-dessus. Aucun n'est exhaustif, car les techniques en question sont extrêmement nombreuses et diverses, et les articles sont souvent ciblés sur les systèmes couche/substrat $[3,14-20]$. Nous tenterons cidessous, sans espérer non plus l'exhaustivité, de citer les techniques les plus souvent pratiquées, en les situant par rapport aux deux types d'exigences précédemment définies, et en insistant sur celles qui permettent d'accéder à une mesure quantitative fiable de l'énergie de rupture interfaciale.

\subsection{Techniques de mesure de seuil de décohésion}

On retrouve dans cette catégorie beaucoup d'essais développés pour caractériser les assemblages collés, applicables aux bi-matériaux ou aux systèmes couche/substrat, de même que quelques essais plus spécifiques des interfaces solide/solide (Fig. 3) :

- cisaillement à simple ou double recouvrement (« shearlap » test) ;

- cisaillement sur barreau revêtu (essai Ollard, « rond de serviette $\gg)$;

- torsion de tube bi-matériau;

- «push test $»$;

- « pull test » ou arrachement par pion collé;
- cisaillement bi-entaillé;

- guillotine.

Dans le cas des couches sur substrats, diverses variantes de l'essai de pelage permettent de mesurer soit des seuils d'effort, soit des fractions d'interfaces décollées (Fig. 3). Toujours dans le cas des revêtements, l'essai de rayage sous charge croissante (« scratch test ») mérite également une mention particulière : très utilisé, il ne nécessite pas de préparation particulière de l'échantillon; instrumenté à l'aide d'un capteur acoustique, il permet de détecter la valeur critique de l'effort normal qui provoque les premiers écaillages du revêtement en bordure du sillon de rayure (Fig. 3).

\subsection{Techniques permettant la mesure en continu de l'énergie de rupture interfaciale}

Pour tous les essais de cette catégorie, des modélisations ont été développées pour permettre d'aboutir à une valeur numérique de l'énergie de rupture interfaciale. Tous opèrent sur des éprouvettes pré-fissurées et tous satisfont aux critères définis à la section 3.1.2 : les pré-fissurations sont généralement obtenues soit par des moyens mécaniques, soit par contamination d'une partie de l'interface lors de son élaboration. Outre des éprouvettes classiquement utilisées en mécanique de la rupture de matériaux homogènes, on y retrouve des essais développés pour tester la résistance au délaminage des composites stratifiés ou des assemblages collés (Fig. 4) :

- « Double Cantilever Beam » (DCB);

- « End-Notched Flexure » (ENF);

- « End Loaded Split » (ELS);

- décollement par un revêtement en tension (« Edge Lift-off $»)$;

- décollement par poinçon («point-blister test »);

- flexion 4-points en mode normal;

- flexion 4-points en mode mixte;

- pelage en $\mathrm{T}$;

- clivage en coin («Edge test, blade test $»)$;

- pelage sur rouleau;

- gonflement-décollement (cf. Sect. 4).

Une mention particulière est à faire pour l'essai de flexion 4-points asymétrique (ou sa variante, l'essai Iosipescu), l'essai brésilien et l'essai Arcan (Fig. 4), opérant sur des éprouvettes massives, qui sont les trois seuls essais permettant de faire varier la mixité modale de manière continue sur un même type d'éprouvettes (outre des montages spécifiques d'essai biaxial comme celui développé par Liechti et Chai [5]).

\subsection{Techniques permettant un bilan d'énergie d'amorçage et de rupture}

Signalons également qu'il existe quelques essais intermédiaires entre les deux catégories précédentes, qui, partant d'un échantillon intact, permettent un bilan de 

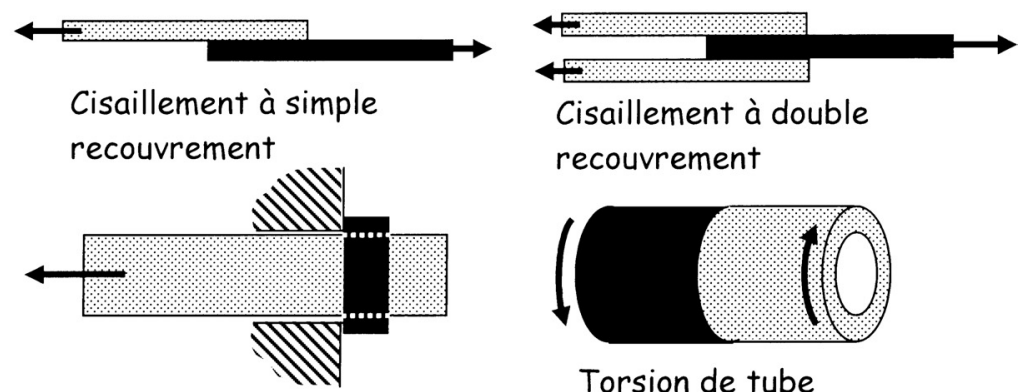

"Rond de serviette"
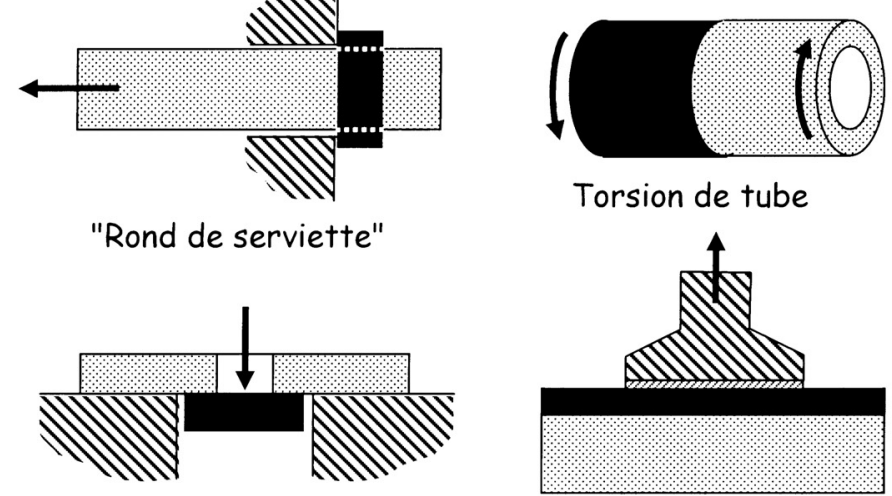

Torsion de tube

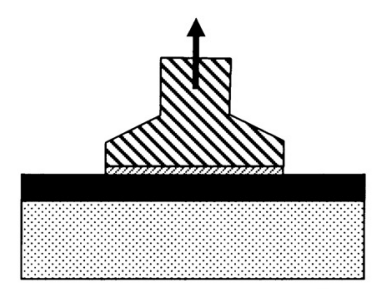

Push test

Pull test, ou pion collé
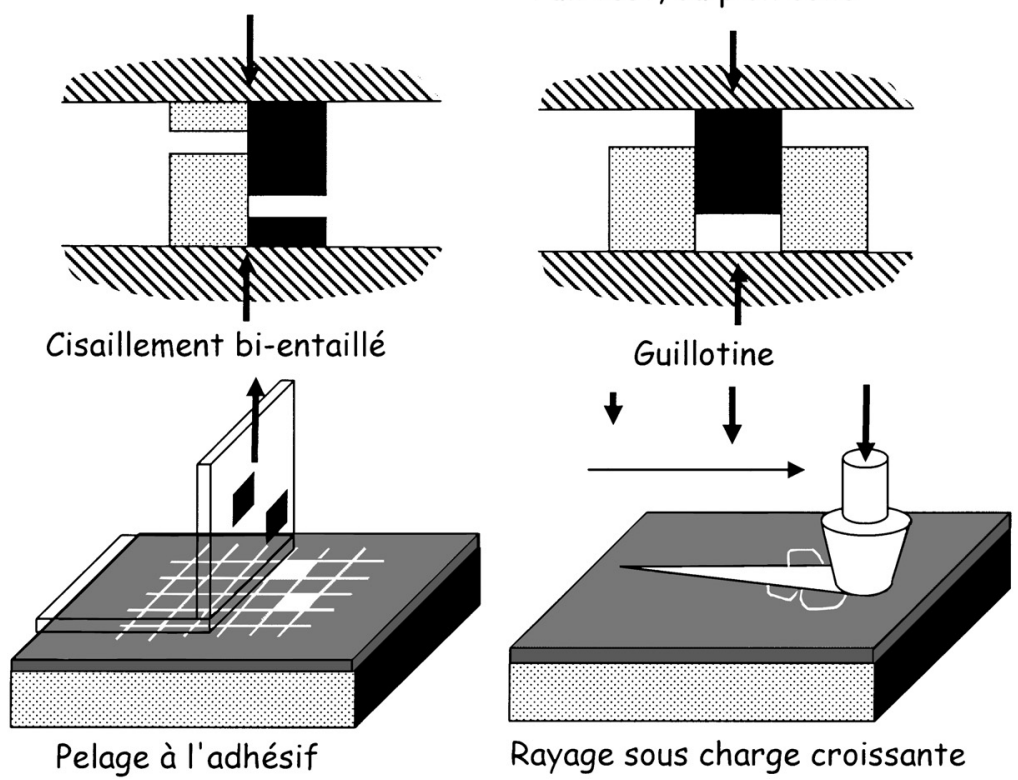

Pelage à l'adhésif

Rayage sous charge croissante

Fig. 3. Représentation schématique de quelques essais de mesure de seuil de décohésion.

l'énergie nécessaire pour amorcer et propager une fissure de dimensions données. Les énergies ainsi obtenues, résultats d'une simple comparaison état initial/état final, constituent naturellement des informations quantitatives précieuses à défaut de mieux, mais présentent l'inconvénient d'incorporer le travail nécessité par l'amorçage de la fissure (souvent aléatoire comme nous l'avons déjà signalé), et d'ignorer le plus souvent les conditions mécaniques exactes tant d'amorçage que de propagation de la fissure interfaciale, observée uniquement à l'état final (notamment la mixité modale). On peut mentionner, dans cette catégorie d'essais (Fig. 5) :

- indentation normale de couches minces;

- indentation interfaciale de couches épaisses ou interfaces massives ;

- flexion ou traction-flambage de revêtements fragiles sur substrats ductiles;

- écaillage par impulsion laser sur couches minces.

\section{Exemple de l'essai de gonflement-décollement}

\subsection{Principe de l'essai de gonflement-décollement}

L'essai de gonflement-décollement est applicable à un revêtement ductile sur substrat. Il consiste à injecter un fluide incompressible sur la face arrière du revêtement, à travers un orifice ménagé dans le substrat par une technique appropriée [21]. Sous l'effet du fluide, dans un premier temps la membrane autoportante constituée par le revêtement se gonfle, avec une flèche maximale $h$ qui est une fonction croissante du volume injecté et de la pression du fluide (Fig. 6a). À partir d'une pression suffisante pour provoquer une tension critique sur le pourtour de la membrane, un décollement de celle-ci de son substrat est amorcé, puis propagé (Fig. 6b). Il en résulte une augmentation rapide du volume inscrit sous la cloque, 


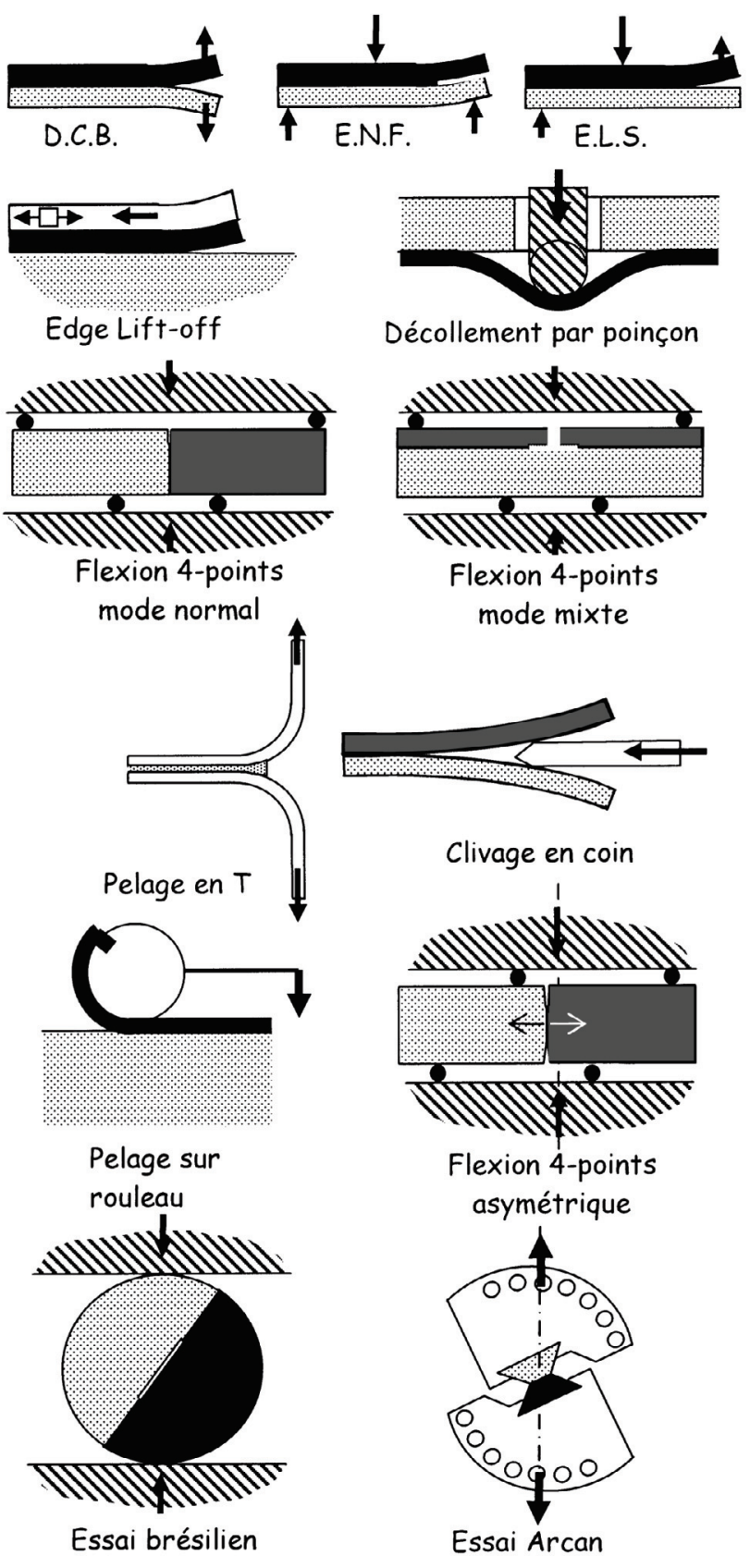

Fig. 4. Représentation schématique de quelques essais de mesure d'énergie de rupture interfaciale.

qui se traduit par une relaxation de la pression (d'où une stabilité intrinsèque de la propagation en régime d'injection volumétrique). L'enregistrement de la pression appliquée $p$ en fonction de la flèche $h$ de la membrane fait clairement apparaître ces deux étapes (exemple Fig. 7).

Dans le cas d'une membrane à comportement élastique, la courbe croissante de gonflement est décrite par l'équation ci-dessous qui comporte trois termes, décrivant respectivement l'effet d'une éventuelle contrainte résiduelle $\sigma_{0}$ dans la membrane, l'effet de la rigidité de la membrane à la traction biaxiale, et l'effet de sa rigidité à la flexion sur les bords de la fenêtre :

$$
p=C_{1} \sigma_{0} \frac{t}{a^{2}} h+C_{2} \frac{E}{(1-\nu)} \frac{t}{a^{4}} h^{3}+C_{3} \frac{E}{\left(1-v^{2}\right)} \frac{t^{3}}{a^{4}} h
$$

Dans cette équation, $E$ et $\nu$ désignent le module de Young et le coefficient de Poisson du matériau constituant la membrane; $C_{1}, C_{2}$ et $C_{3}$ sont des constantes adimensionnelles connues à partir de calculs par la théorie des membranes élastiques. Un ajustement numérique sur des courbes de gonflement permet donc de déterminer les 


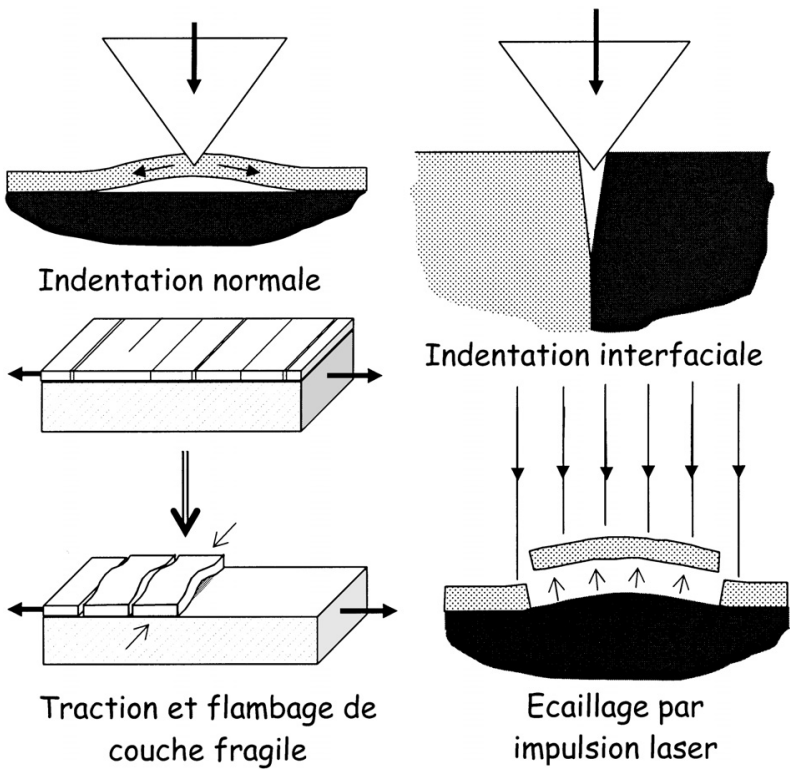

Fig. 5. Représentation schématique de quelques essais de bilan d'énergie d'amorçage et de rupture interfaciale.

a)

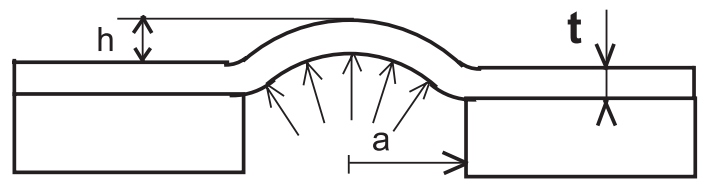

$\mathrm{p}$

b)

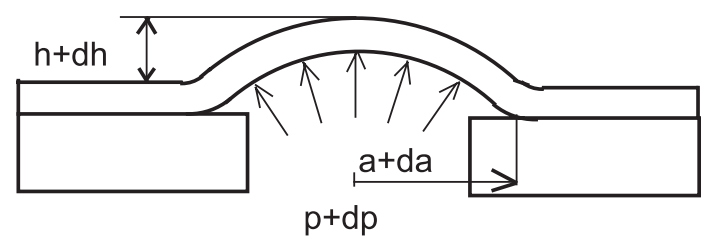

Fig. 6. Schéma des deux étapes de l'essai de gonflementdécollement de revêtement sur substrat : a) gonflement; b) décollement.

valeurs de $\sigma_{0}, E$ et $\nu$ sur une membrane de géométrie connue.

La courbe décroissante de décollement peut être modélisée en écrivant le bilan de l'énergie mécanique apportée au système par le fluide sous pression, consommée d'une part en énergie de déformation de la membrane, d'autre part en croissance de la fissure interfaciale à raison de $G_{i c}$ par unité d'aire. Toujours dans le cas d'un comportement élastique, ceci se traduit par l'équation hyperbolique (11), où $C$ est une constante adimensionnelle voisine de 0,5 :

$$
\text { C.p.h }=G_{i c}
$$

Un ajustement numérique de la courbe expérimentale de décollement permet donc une mesure directe et continue de $G_{i c}$, énergie mécanique d'adhérence interfaciale du revêtement sur son substrat, sous une mixité modale connue et stationnaire, généralement de l'ordre de $-55^{\circ}$.

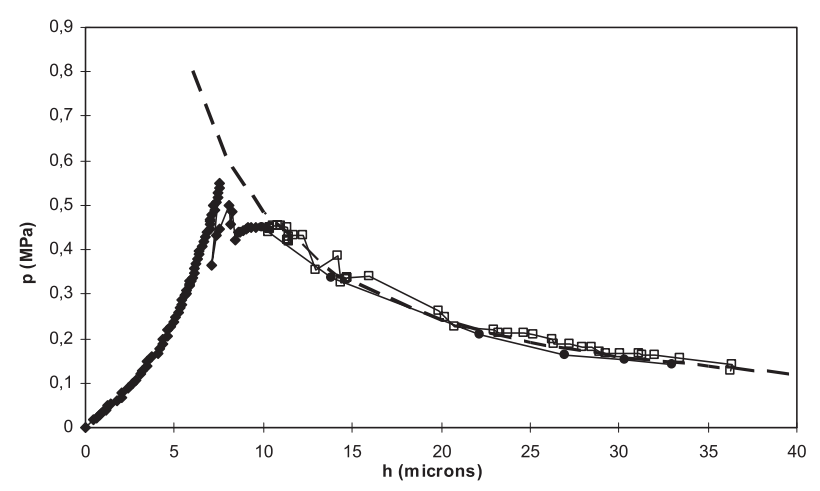

Fig. 7. Exemple de courbe de gonflement-décollement d'un échantillon $\mathrm{W} / \mathrm{SiO}_{1,24} \mathrm{~N}_{0,63} / \mathrm{Si}$. La courbe en pointillés est l'hyperbole d'équation C.p.h $=2,4 \mathrm{~J} . \mathrm{m}^{-2}$.

Nous avons mis en œuvre cet essai à l'aide d'un montage dans lequel l'échantillon, après sa préparation, est bridé sur la platine d'un bloc de pressurisation muni d'un manomètre et d'un pousse-seringue permettant d'injecter de l'eau distillée avec un débit réglable. Un dispositif de profilométrie optique par projection de franges permet une acquisition instantanée de l'ensemble de la géométrie de la cloque. Grâce à un utilitaire développé sous Labview ${ }^{\circledR}$, un PC-maitre peut piloter l'injection du fluide de pressurisation, le déclenchement des acquisitions de profilométrie et le relevé simultané de la pression appliquée, à des intervalles de temps réguliers.

\subsection{Système $\mathrm{W} / \mathrm{SiO}_{2} / \mathrm{Si}$}

Des dépôts de tungstène de 1 à $4 \mu \mathrm{m}$ d'épaisseur ont été effectués par pulvérisation cathodique magnétron sur des plaques monocristallines de silicium (100), revêtues d'une sous-couche PECVD dont la composition était choisie entre la silice $\mathrm{SiO}_{2}$ pure et le nitrure de silicium $\mathrm{Si}_{3} \mathrm{~N}_{4}$, en passant par deux oxynitrures intermédiaires. Des opérations de photolithographie et de gravure chimique anisotrope ont permis de perforer le substrat de silicium et la sous-couche pour pouvoir appliquer la pression d'eau distillée sur la face inférieure du film de tungstène [22]. Les courbes de gonflement et décollement obtenues sur ces échantillons, dont le comportement est parfaitement élastique, sont l'illustration exacte des équations (10) et (11), comme le montre la figure 7 .

Les valeurs d'énergie de propagation des fissures interfaciales issues de cette série d'expériences révèlent que l'énergie d'adhérence du tungstène sur sa sous-couche est une fonction croissante de la teneur en azote de celleci, depuis $0,7 \mathrm{~J} . \mathrm{m}^{-2}$ pour l'interface $\mathrm{W} / \mathrm{SiO}_{2}$, jusqu'à $4,1 \mathrm{~J} \cdot \mathrm{m}^{-2}$ pour $\mathrm{W} / \mathrm{Si}_{3} \mathrm{~N}_{4}$.

\subsection{Système résine époxyde/acier inoxydable}

Nous avons préparé des substrats d'épaisseur $1 \mathrm{~mm}$ en tôle d'acier inoxydable AISI 304/EN X6CrNi18-9, sous forme de carrés de $17 \mathrm{~mm} \times 17 \mathrm{~mm}$, percés en leur centre 


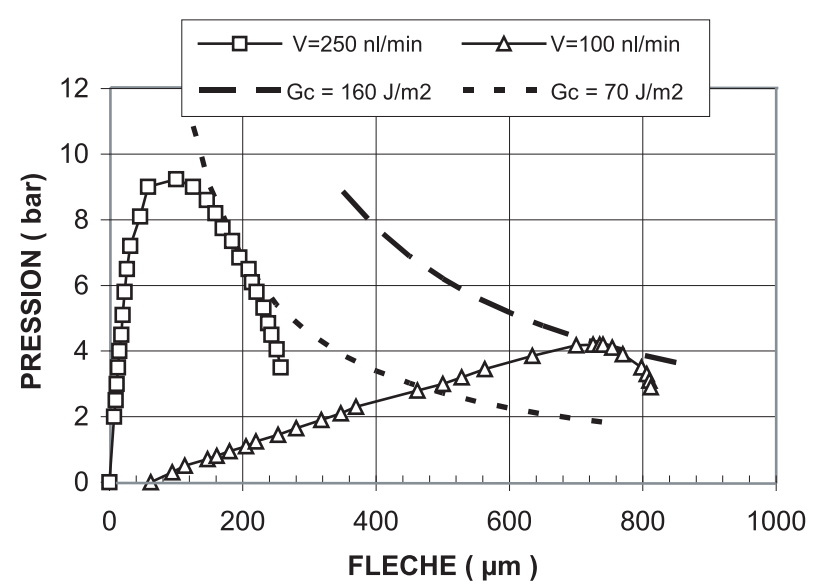

Fig. 8. Gonflement-décollement de membranes de résine époxyde CIBA $2016^{\circledR}$ sur acier 304.

d'un trou de diamètre $1 \mathrm{~mm}$. Cet orifice est masqué par un opercule de ruban adhésif mince sur la face utile de l'échantillon, qui est ensuite revêtue d'une couche de résine époxyde d'épaisseur variable mais contrôlée, entre 100 et $500 \mu \mathrm{m}$. Deux résines ont été utilisées : CIBA $2016^{\circledR}\left(T_{\mathrm{g}} \cong 30{ }^{\circ} \mathrm{C}\right)$ et $\mathrm{CIBA} 2015^{\circledR}\left(T_{\mathrm{g}} \cong 75^{\circ} \mathrm{C}\right)$, étuvées après étalement dans les conditions préconisées par le fabricant.

Les courbes de gonflement-décollement obtenues sur ce système s'écartent clairement du comportement purement élastique décrit par les équations (10) et (11), en raison du fluage visco-élastique des résines époxydes. Pendant le stade de gonflement, ce fluage entraîne une augmentation de la flèche $h$ qui s'amplifie au cours du temps. Pendant le stade de décollement, le fluage de la membrane amplifie l'effet de relaxation de la pression : de ce fait, l'ensemble du décollement ne suit généralement pas la loi hyperbolique (11) qui n'est plus valable que localement, pour indiquer l'énergie d'adhérence consommée qui varie ainsi au cours du décollement.

La valeur du débit d'injection commande non seulement la vitesse de gonflement, mais aussi la vitesse de propagation du front de fissure pendant le décollement. On peut voir son influence sur la figure 8 : un débit d'injection faible permet à la membrane de fluer beaucoup plus pendant le gonflement, et d'atteindre ainsi des déflexions élevées. Bien que la pression de début de décollement soit plus faible, on constate sur l'exemple de la figure 8 que l'énergie maximale consommée lors du décollement (repérée par le niveau de l'hyperbole tangente à la courbe de décollement) est plus de deux fois supérieure à celle du décollement avec le débit le plus élevé.

La comparaison - toutes choses égales par ailleurs entre les énergies d'adhérence maximales des résines sur substrats acier inoxydable 304 à l'état 2R (recuit brillant), de très faible rugosité, et à l'état $2 \mathrm{~B}$ (recuit décapé), à rugosité plus importante, fait apparaître un écart d'environ $20 \%$ en faveur de l'état recuit décapé. Cette augmentation est imputable aux effets d'ancrage mécanique de la résine dans les sillons gravés aux joints des grains du métal sur la surface décapée.

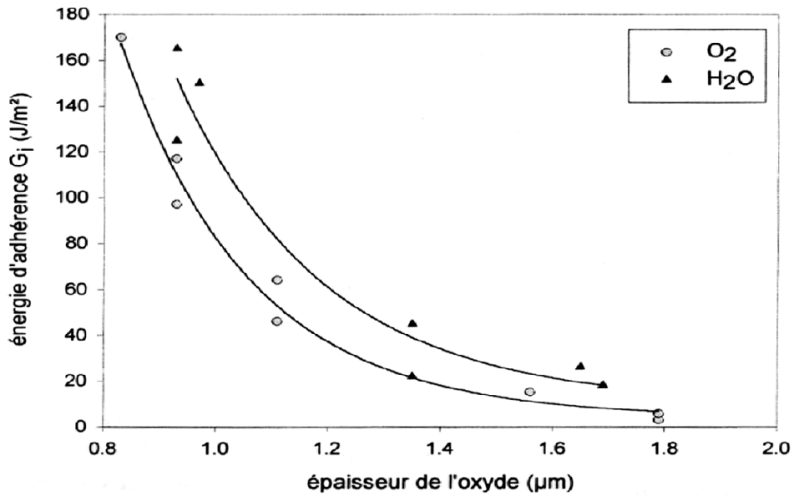

Fig. 9. Énergie d'adhérence Fe-18 Cr-Ti Nb/oxyde thermique en fonction de l'épaisseur de la couche d'oxyde, en atmosphère d'oxygène $\left(\mathrm{O}_{2}\right)$ ou de vapeur d'eau $\left(\mathrm{H}_{2} \mathrm{O}\right)$.

Les effets de fluage visqueux sont nettement plus prononcés avec les revêtements de résine CIBA $2016^{\circledR}$, dont la température de transition vitreuse est proche de l'ambiante, qu'avec la résine CIBA $2015^{\circledR}$.

\subsection{Système acier inoxydable/oxyde thermique}

Compte tenu de la fragilité intrinsèque d'une couche d'oxyde thermique à la surface de son métal de base, il ne saurait être question de tenter de la décoller par gonflement direct. Dans ce cas, nous avons eu recours à des échantillons en position dite «inversée » : un feuillard métallique (en l'occurrence un acier inoxydable ferritique de type $\mathrm{Fe}-18 \mathrm{Cr}-\mathrm{Ti} \mathrm{Nb}$ ) recouvert sur les deux faces de sa couche d'oxyde thermique (d'épaisseur comprise entre 0,8 et $2 \mu \mathrm{m}$, générée à $900{ }^{\circ} \mathrm{C}$ en atmosphère d'oxygène ou de vapeur d'eau), est collé sur le porte-échantillon par un adhésif structural cyanoacrylate. Sous l'effet de la pression d'eau distillée, c'est le feuillard métallique qui se décolle de sa couche d'oxyde, qui demeure sur le porteéchantillon.

Compte tenu de la relative rigidité du feuillard métallique, le troisième terme de l'équation (10) est prépondérant, et les courbes de gonflement sont pratiquement linéaires en coordonnées $p \times h$. Une procédure comportant plusieurs gonflements et décollements successifs sur le même échantillon a permis d'atteindre un encadrement de l'énergie d'adhérence à $\pm 2 \mathrm{~J} \mathrm{~m}^{-2}$ près. Le résultat le plus marquant de cette étude est la chute très rapide de l'énergie d'adhérence métal/oxyde lorsque la couche d'oxyde s'épaissit (Fig. 9), en raison de différents mécanismes interfaciaux de perte d'adhérence, que l'examen au Microscope Électronique à Balayage des surfaces décollées permet d'identifier [23].

\section{Conclusions}

Des techniques efficaces existent pour déterminer l'énergie mécanique d'adhérence des interfaces solides. Leur application demande des précautions et des 
modélisations fiables. Abstraction faite des problèmes d'amorçage de fissure, le paramètre qui semble le plus pertinent en la matière est l'énergie de propagation des fissures interfaciales, assortie impérativement de la connaissance de la mixité modale sous laquelle la fissure s'est propagée.

La limite - tant pratique que théorique - des techniques de mesure d'adhérence est atteinte lorsque i) la taille des interfaces à tester rend difficile la préparation d'éprouvettes adéquates (problèmes des microsystèmes, des interfaces précipités/matrices, etc.); ii) l'un des matériaux testés est l'objet d'une entrée en plasticité généralisée, qui fait dépendre l'énergie mesurée de la taille des échantillons; iii) l'énergie de rupture de l'interface s'élève au même ordre de grandeur que l'énergie de rupture cohésive de l'un des deux matériaux adjacents - l'utilisateur pourra sans doute facilement admettre alors que l'adhérence est suffisante pour l'interface considérée...

Remerciements. Cet article doit beaucoup à de multiples discussions avec A. Bosseboeuf (IEF, Université Paris-Sud), D. Leguillon (LMM, Université Paris-VI) et J. Laurencin (CEA-Grenoble) au cours des années passées. Le dispositif de gonflement-décollement présenté a été mis en place et développé avec le soutien du GDR « Caractérisation des Interfaces dans les Multimatériaux »du CNRS, jusqu'en 1997, puis avec l'aide matérielle et scientifique du Centre de Recherches d'Ugine du groupe ARCELOR et d'une ACI Surfaces et Interfaces « Adhérence Epoxy/inox ».

\section{Références}

[1] Revue de Métallurgie, Science et Génie des Matériaux, Numéro spécial sur le thème Adhésion, adhérence, $\mathrm{N}^{\circ} 5$, Mai 1997

[2] J.L. Chaboche, R. Girard, P. Levasseur, On the interface debonding models, Int. J. Damage Mechanics 6 (1997) $220-257$

[3] H.C. Cao, A.G. Evans, An experimental study of fracture resistance of bimaterial interfaces, Mechanics of Materials 7 (1989) 295-304

[4] J.S. Wang, Z. Suo, Experimental determination of interfacial toughness curves using brazil-nut-sandwiches, Acta Metal. Mater. 38 (1990) 1279-1290

[5] K.M. Liechti, Y.S. Chai, Asymmetric shielding in interfacial fracture under in-plane shear, J. Appl. Mechanics 59 (1992) 295-304

[6] N.P. O'Dowd, M.G. Stout, C.F. Shih, Fracture toughness of alumina-niobium interfaces: experiments and analyses, Phil. Mag. A 66 (1992) 1037-1064

[7] J. Dundurs, Discussion on: Edge-bonded dissimilar orthogonal elastic wedges under normal and shear loading, D.B. Bogy (ed.), J. Appl. Mechanics 36 (1969) 650-652

[8] C.F. Shih, Cracks on bimaterial interfaces: elasticity and plasticity aspects, Mat. Sci. Eng. A 143 (1991) 77-90
[9] D. Leguillon, S. Bein, M. Dupeux, J. Frelat, Mixité modale pour une fissure d'interface; application à l'essai brésilien, Revue Européenne des Éléments Finis 9(1-2-3) (2000) 263-276

[10] J. Laurencin, M. Dupeux, D. Leguillon, Amorçage et propagation d'une fissure à l'interface d'un bi-matériau, Communication au Colloque de l'École de Mécanique Mecamat - Aussois 2002, Mécanismes et déformations dans les matériaux multiconstituants, Aussois, 21-25 Janvier 2002

[11] A.K. Chouaf, G. Loppin, M. Ignat, J.M. Terriez, Analysis of stresses at singular points of patterned structures, Mat. Res. Soc. Symp. Proc. 309 (1993) 211-216

[12] D. Leguillon, K. Siruguet, Finite fracture mechanics Application to the onset of a crack at a bimaterial corner, Proc. IUTAM Symp. on Analytical and computational fracture mechanics of non-homogeneous materials, Cardiff (UK), 18-22 June 2001, B.L. Karihaloo (ed.), Kluwer Acad. Press, Dordrecht, Germany, pp. 11-18

[13] D. Leguillon, Strength or toughness? A criterion for crack onset at a notch, Eur. J Mech. A/Solids 21 (2002) 61-72

[14] J.E. Pawel, C.J. McHargue, Testing of adhesion of thin films to substrates, J. Adhesion Sci. Technol. 2(5) (1988) 369-383

[15] D.S. Rickerby, A review of the methods for the measurement of coating-substrate adhesion, Surf. Coatings Technol. 36 (1988) 541-557

[16] P.A. Steinmann, H.E. Hintermann, A review of the mechanical tests for assessment of thin film adhesion, J. Vac. Sci. Technol. A7(3) (1989) 2267-2272

[17] N.P. O'Dowd, C.F. Shih, M.G. Stout, Test geometries for measuring interfacial fracture toughness, Int. J. Solids Structures 29(5) (1992) 571-589

[18] C.K. Lin, C.C. Berndt, Measurement and analysis of adhesion strength for thermally sprayed coatings, J. Thermal Spray Technol. 3(1) (1994) 75-104

[19] H. Weiss, Adhesion of advanced overlay coatings : mechanisms and quantitative assessment, Surf. Coatings Technol. 71 (1995) 201-207

[20] A.A. Volinsky, N.R. Moody, W.W. Gerberich, Interfacial toughness measurements for thin films on substrates, Acta Mater. 50 (2002) 441-466

[21] A. Bosseboeuf, M. Dupeux, M. Boutry, T. Bourouina, Bulge and Blister Test for Assessment of Coating Strength and Interfacial Adhesion: Experimental Challenge and Theoretical Tools, Proc. Int. Workshop on Mechanical behaviour of P.V.D. Coated Materials, 13-17 Oct. 1997, Holzau/Erzgebirge (All.), Techn. Univ. Bergakademie Freiberg, 1998, pp. 169-181

[22] M. Dupeux, A. Bosseboeuf, T. Bourouina, D. Buttard, Investigation of the Mechanical Properties and Adhesion of P.V.D. Tungsten Films on Si and Silicon Compounds by Bulge and Blister tests, Mat. Res. Soc. Symp. Proc. Ser. 518 (1998) 87-92

[23] J. Mougin, M. Dupeux, A. Galerie, L. Antoni, Study of the adhesion of thermal oxide layers grown on several ferritic stainless steel using the inverted blister test, Mater. Sci. Technol. 18 (2002) 1217-1220 